\title{
Direct and indirect effects of action on object classification
}

\author{
EUN YOUNG YOON and GLYN W. HUMPHREYS \\ University of Birmingham, Birmingham, England
}

\begin{abstract}
We report three experiments in which name verification responses to either objects (Experiments 1 and 2) or hand movements (Experiment 3) were compared with action decisions, where participants verified whether an object is typically used in the way described by a verbal label. In Experiments 1 and 2, we report that action decisions show more consistent and larger effects of the congruency of either a handgrip or a type of movement than do name verification responses, although there was some effect of the congruency of the handgrip on name verification. In Experiment 3, we demonstrate that the congruency of the object being moved affects both action and name verification responses to hand movements. We discuss the data relative to accounts of how actions and names are accessed by visually presented objects and in relation to work on the information called upon in classification tasks.
\end{abstract}

\section{Dual Routes to Action}

In our everyday lives, we frequently perform actions that are directed at the objects that surround us. These actions involve online guidance of our effectors in response to objects, plus the selection of the appropriate category to which the action belongs (e.g., drinking from a cup, rather than using it to brush a crumb from a table). This article is concerned with how such categories of action are selected for visually presented objects. Traditionally, theories have stressed that the retrieval of an action is guided by access to semantic knowledge based on an object's associations and its abstract function (e.g., Ochipa, Rothi, \& Heilman, 1992; Roy \& Square, 1985). For instance, a cup activates the action of drinking through access to semantic knowledge based on our prior associations with how cups are used and what they are used for. However, there is also increasing neuropsychological and experimental evidence that action is evoked by the visual properties of objects in a relatively direct way, without the necessary involvement of semantic (associative) memory (see Humphreys, 2001, and Humphreys \& Riddoch, 2003, for reviews; also see Barsalou, 1999, for a similar view derived from a different literature). This evidence provides the backdrop for the present study, in which some of the properties of a putative direct route to action from vision were examined.

Neuropsychological evidence. There is considerable neuropsychological evidence for a relatively direct influence of the visual properties of objects when actions are made to them, as compared with cases in which semantic information is retrieved. This evidence comes primarily

This work was supported by an ESRC grant to the first author and by a Wellcome Trust project grant and an MRC program grant to the second author. Correspondence concerning this article should be addressed to E.Y. Yoon, Behavioural Brain Science Centre, School of Psychology, University of Birmingham, Birmingham B15 2TT, England (e-mail: e.y.yoon@bham.ac.uk). from studies of the syndromes of optic aphasia, semantic dementia, and apraxia. Optic aphasia is characterized as an apparently modality-specific naming disorder in which patients are poor at naming visually presented objects, as compared with when they name definitions (e.g., Beauvois, 1982; Coslett \& Saffran, 1992; Hillis \& Caramazza, 1995; Lhermitte \& Beauvois, 1973; Manning \& Campbell, 1992; Riddoch \& Humphreys, 1987). Despite their poor naming performance, such patients are relatively good at making appropriate gestures to objects. Such gestures have usually been interpreted as indicating spared recognition. However, detailed analyses have suggested that visual recognition (and access to semantic knowledge) can be disrupted. For example, J.B., the patient reported by Riddoch and Humphreys, was impaired at matching associatively related objects (e.g., hammer and nail vs. wrench). In contrast to his poor matching performance with vision, J.B. could match items from their names. Thus, semantic knowledge (from names) was relatively intact, but there was poor access to this knowledge with vision. To account for J.B.'s good gesturing to visually presented objects, Riddoch and Humphreys proposed that his gestures could be based on direct associations between objects and actions, which operated despite his poor access to semantics from objects. A framework by which to account for J.B.'s case (and the other cases discussed below) is provided in Figure 1. In Figure 1, J.B.'s deficits would follow from a lesion at Location A. Similar results have been reported by Hillis and Caramazza (1995).

Other neuropsychological evidence for direct visual influence on actions directed at objects has come from studies of semantic dementia. Semantic dementia patients can have degenerative conceptual knowledge about objects (Snowden, Goulding, \& Neary, 1989). Hodges, Bozeat, Lambon Ralph, Patterson, and Spatt (2000) reported that patients with semantic dementia are impaired on object use, with the magnitude of any deficit being correlated 


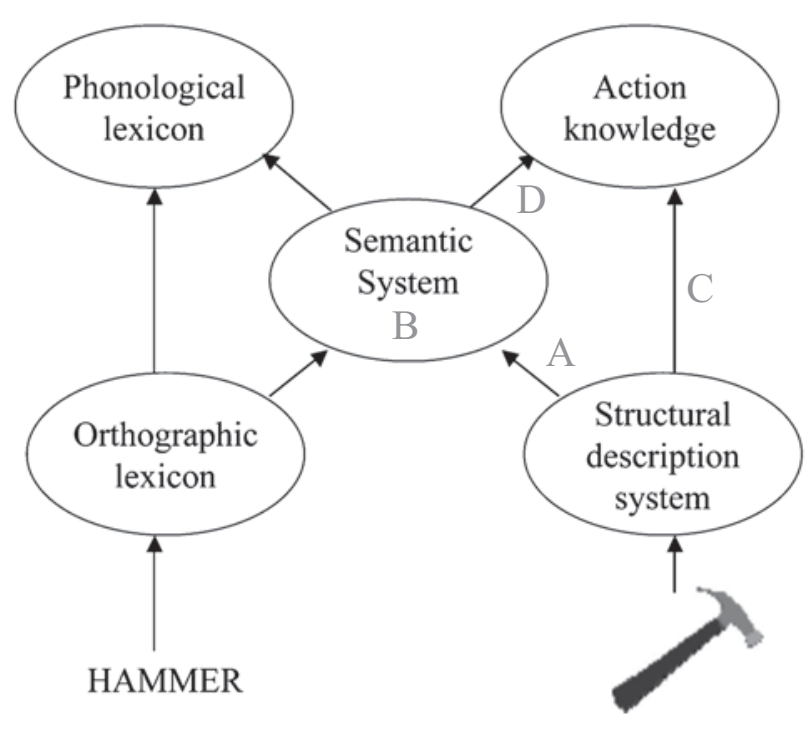

Figure 1. The framework of the dual-route model (adapted from Riddoch, Humphreys, \& Price, 1989), along with putative sites of damage in the neuropsychological syndromes of (A) optic aphasia, (B) semantic dementia, and (C) visual apraxia (see the text for details). From "Routes to Action: Evidence From Apraxia," by M. J. Riddoch, G. W. Humphreys, and C. J. Price, 1989, Cognitive Neuropsychology, 6, p. 439. Copyright 1989 by Taylor and Francis Group. Adapted with permission.

with the degree of semantic knowledge loss. From this, they suggested that object use depends on access to conceptual knowledge. Nevertheless, Hodges, Spatt, and Patterson (1999) reported that individual patients with semantic degradation can perform relatively normally when asked to make judgments about how to use novel tools, despite their deficit in semantic knowledge. Cases of preserved object use can even extend to multistep actions. Riddoch, Humphreys, Heslop, and Castermans (2002) documented Patient M.C., who performed well on everyday tasks with common objects, making fewer errors than did a group of control dementia patients. Despite this, M.C. was worse than the dementia controls at making superordinate categorization decisions (would you find this in the garden?) and at providing definitions of objects. Riddoch et al. (2002) suggested that her everyday actions were supported by direct (nonsemantic) links between vision and action. In terms of Figure 1, semantic dementia can be linked to a lesion at Location B.

A pattern of performance opposite to that of optic aphasic and semantic dementia patients has been shown by patients with visual apraxia, who are impaired in their ability to gesture at visually presented objects (see, e.g., DeRenzi, Faglioni, \& Sorgato, 1982; Pilgrim \& Humphreys, 1991; Riddoch, Humphreys, \& Price, 1989). This is not simply a motor disorder, since the patients can gesture at the names of objects. In fact, visual apraxics can also have intact object recognition and object naming, so that the impaired actions for objects cannot be attributed to poor semantic access from vision. To account for this pattern of results, Riddoch et al. (1989) proposed that damage to a direct visual route "blocked" an intact semantic route for action (evident when the patients gestured to object names; see Figure 1, Lesion Location C). Yoon, Heinke, and Humphreys (2002) have modeled these patterns of impairment in terms of a convergent route model of action. In this model, activation from separate semantic and visual routes converges to drive the retrieval of appropriate categories of actions for objects. In visual apraxia, noise, created by a lesion within the visual route, can "push" activation away from the stable state that represents the learned categorical action for the object. ${ }^{1}$ Since the direct route has an input for action, but not for object names, selective damage to the visual route can affect action retrieval more than it affects name retrieval for objects. Also, in visual apraxia, action retrieval from words is consistent with a relatively undamaged (Riddoch et al., 1989) route from semantics to action, which is relatively preserved (Location D, Figure 1).

Experimental evidence. Rumiati and Humphreys (1998) examined the types of errors made by normal participants when they were required either to make gestures at or to name pictures and words presented under deadline conditions (the deadline was used to induce a relatively high error rate). When gestures at pictures were required, the participants tended to make relatively high proportions of visual errors (e.g., making a writing action for a toothbrush; this was termed a visual error because a toothbrush is similar to a pen in shape, but not in function). In contrast, errors in naming under deadline conditions tended to be semantic rather than visual (toothbrush $\rightarrow$ toothpaste; note that toothpaste is associatively, but not visually, related to a toothbrush). Similarly, gesturing at words tended to produce semantic errors. Rumiati and Humphreys suggested that the generation of these different types of errors was contingent on the dual routes to action from vision. For objects, a visual route led to visual errors being generated, under deadline conditions, since the responses were based on information that was made available most rapidly to response systems (direct visual, rather than semantic). Semantic errors were found in object naming and gesturing to words, since these tasks were mediated by access to semantic knowledge.

Chainay and Humphreys (2002) have provided converging evidence for the distinction between semantic knowledge and knowledge about how objects are used in action. They had participants make two types of decisions with respect to objects and to words corresponding to the objects. For one type of decision, the participants decided whether the object would typically be found in a kitchen, a task assumed to depend on access to contextual knowledge in the semantic system. For the other, the participants made a decision about how the object would be used: Would a twisting or a pouring action be employed (an action decision)? Chainay and Humphreys found little difference in the times required to make semantic decisions for objects and words, whereas action decisions were faster for objects than for words. These 
faster decisions for objects were not caused by the presence of diagnostic features, since action decisions were delayed for nonobjects that contained the same features as the objects. Chainay and Humphreys proposed that access to contextual semantic knowledge was equally fast for objects and words. In contrast, only objects could activate action knowledge directly, whereas action knowledge for words was accessed through the semantic system. Fast, direct access to action knowledge facilitated action decisions for objects.

It is interesting that in the studies of Chainay and Humphreys (2002) and Rumiati and Humphreys (1998), evidence for a direct route to action knowledge arose with pictorial stimuli and when actions did not have to be made to objects. Apparently, action knowledge can be evoked by the structural properties of objects depicted in line drawings, as well by real three-dimensional objects. We also note that an advantage for accessing action knowledge over semantic contextual knowledge arose even when explicit actions were not made to the objects. It may be that representations involved in the motor actions to the objects were invoked and these contributed to the effects. It is clear, though, that actual actions are not necessary.

\section{The Nature of the Dual Routes to Action}

Although the evidence above is consistent with visual information being used to access categories of action directly, the nature of the visual information involved remains poorly understood. One factor that seems to be important is the orientation of the object, since object orientation seems to play a more critical role in retrieving actions than in retrieving semantic/associative knowledge about objects.

Humphreys and Riddoch (2001) reported a patient with spatial neglect, M.P., who was impaired at detecting visual objects defined by their name (e.g., find the cup) when they fell on the side of space contralateral to his lesion. Nevertheless, his detection was enhanced when the objects were cued by their associated action (find the objects to drink out of ). This effect was found even with nonobjects, suggesting that it was contingent on actions that were afforded by the visual properties of objects, without knowledge of individual instances. However, the effect broke down if the objects were oriented away from the patient so that they could not be directly linked to action. In contrast, the same orientation changes had little effect on recognition, which was indicated by M.P.'s ability to find objects from their names. Humphreys and Riddoch (2001) suggested that the patient continued to be able to use information carried by a direct (nonsemantic) route to action but that this route was sensitive to object orientation.

Other neuropsychological evidence for an influence of object orientation on action retrieval comes from studies of utilization behavior in patients with cortico-basal degeneration and/or frontal lobe damage. In the studies of Riddoch, Edwards, Humphreys, West, and Heafield (1998; Riddoch, Humphreys, \& Edwards, 2000), patients were required to reach for and grasp a cup placed on either the left or the right side of a table, using the hand aligned with the cup, irrespective of whether the handle of the cup was oriented to the right or the left. The performance of the patients was affected by the orientation of the handle of the cup. For example, right-hand reaches were made to a cup on the left of the patient's body if the handle of the cup was oriented to the right. These errors were involuntary. In fact, the patient described by Riddoch et al. (1998) could not make contralateral reaches when instructed to do so but made such reaches only when they were evoked by the object. Actions seem to be generated in response to the visual properties of objects, and this process is sensitive to viewpoint (the left-right orientation of the cups). In contrast to this, studies of object recognition demonstrate few effects of left-right orientation on naming (Biederman \& Cooper, 1991).

Experimental evidence from normal participants comes from Tucker and Ellis (1998). They demonstrated effects of object orientation on judgments about whether objects were shown in an upright or an inverted orientation. The handles of the objects were turned to either the left or the right, but this was irrelevant to the task. Nevertheless, right-hand responses were facilitated when the handles of the objects were placed to the right, and left-hand responses were facilitated when the handles were placed to the left. Also, priming effects based on orientation were shown by Craighero, Fadifa, Rizzolatti, and Umiltà (1998, 1999). Prime objects whose orientation was congruent with the orientation of the response action facilitated grasping responses to targets. These experimental data suggest that the direct (nonsemantic) route to action is sensitive to object orientation.

Handgrip and hand movements to objects. In this article, we assess whether two other factors, handgrip and hand movements with objects, influence the direct (nonsemantic) route to action and whether the influence on action is greater than any effects on object recognition (access to semantics). To compare responses that weight direct visual information, on the one hand, and semantic information, on the other, we contrasted object name verification and action classification tasks. According to the framework given in Figure 1, name verification for objects is mediated by access to semantic knowledge. This can be contrasted with classifications of whether an object is used to make a particular action (action decisions), which can be based on visual, as well as semantic, information. Hence, for action classification, there should be an influence of the visual properties of objects that are consistent with the action as it is visually performed. When the handgrip and hand movements are congruent with such actions, participants are presented with an instance that fits several of the properties that they witness when viewing the actions of others and when making the actions themselves. Performance should be facilitated, relative to when the object appears alone (the neutral baseline). In contrast, when the handgrip and hand movements are incongruent with the usual action for the object, action 
classification for the object may be impaired, due to the presence of conflicting visual information. Name verification, however, should be less affected by the covarying properties of handgrip and hand movement, because (1) it is contingent on abstracted semantic properties of the object itself (common across different instances of action) and (2) these properties remain the same even when the object is held or moved in an unusual manner.

By using evidence from functional brain imaging particularly, it is possible to argue that any direct route from vision to action is contingent on neural pathways separate from those mediating object recognition. For example, the recognition of many categories of objects seems to depend on activation in the lateral occipital and inferior occipito-temporal cortex (e.g., Chao \& Martin, 2000). An exception to this is the category of tools, where activation is frequently found in the left inferior frontal lobe, the posterior middle temporal gyrus, the posterior parietal cortex, and the MT/MST region linked to motion perception (e.g., Devlin et al., 2002; Grèzes \& Decety, 2002; Kourtzi \& Kanwisher, 2000). Several neuroimaging studies also indicate the involvement of the parietal cortex when participants observe hand actions (e.g., Buccino et al., 2001; Decety et al., 1994; Grafton, Arbib, Fadiga, \& Rizzolatti, 1996), whereas lesions to the parietal cortex can impair action representations for the hand (e.g., Jennerod, Decety, \& Michel, 1994; Sirigu et al., 1996). Hence, these cortical regions may play a role in representing knowledge about how objects are used in action. The fact that these regions can be activated in identification tasks, where actions to the objects are not required, suggests that action associations are evoked even when knowledge of the action is not critical to the task. On the basis of such studies, there are grounds to argue that both handgrip and object motion may influence object identification, whereas even stronger effects may emerge when tasks explicitly require judgments about the actions performed with objects. Note that in the framework illustrated in Figure 1, object identification would depend on access to semantic knowledge, whereas tasks involving action decisions (Chainay \& Humphreys, 2002) may be made by accessing action knowledge directly.

\section{EXPERIMENT 1 STATIC HANDGRIP AND ACTION}

In Experiment 1, the participants carried out action and name decisions for objects that were shown either alone or with a hand adopting a grip that was either congruent or incongruent with how the object is standardly used. ${ }^{2}$ The handgrip, when present, was irrelevant to the decision, which was based on the object alone. We asked whether, nevertheless, the grip affected the speed and accuracy of decision making. If naming is contingent on the retrieval of associative and conceptual knowledge from the shape of the object, it might be affected relatively little by the handgrip. Action decisions, however, may be linked with visual information directly associated with object use and, so, might be affected by the grip. On the other hand, if action decision, like name retrieval, depends on access to semantic knowledge, any effect of the depicted grip might be equivalent across the action and name decision tasks.

\section{Method}

Participants. There were 36 participants, from 18 to 41 years of age. All the participants had normal or corrected-to-normal vision. All except 4 participants reported that they were right-handed.

Materials. Sixty graspable objects were used (see the Appendix for a list of the objects and actions employed): 30 kitchen tools and 30 other manipulable objects. Every object was photographed in three conditions: with a congruent handgrip on the object, with an incongruent handgrip, and with no handgrip (the object alone). Congruent handgrips were the hand configurations that are made when the object is normally grasped for its use. An incongruent handgrip involved adoption of a hand configuration that is inappropriate for the standard use of the object. In the no-handgrip condition, just the objects were presented to the participants. The orientation of the target objects was the same across the conditions (see Figures $2 \mathrm{~A}$ and $2 \mathrm{~B}$ ). The frame size of the stimuli was 450 pixels wide and 370 pixels high $\left(10^{\circ}\right.$ of visual angle), and this window was placed at the center of the computer screen throughout the experiment. An object name or action description was presented in written form simultaneously, just below the photographs. The words were in 20-point Arial font (4.5 of visual angle).

Design and Procedure. There were two experimental tasks: object name and object action verification (see the Appendix for the full instructions). Over both tasks, the participants were required to ignore the handgrips depicted and to focus on the objects. In the object name verification task, the participants had to decide whether the target object matched a name presented below the target image. In the action decision task, the participants were required to verify whether a name presented just below the image of the object described the way the object is usually used. For instance, on a yes trial, the object knife in a picture was paired with the word slicing? (see Figure 2 for further examples). On no trials, the word corresponded to either another name (in name verification) or another action (in action verification), chosen randomly from the other set of names/ action-descriptions used in the study. The participants responded by pressing the keys on the keyboard with either the right or the left hand (half used the right hand to respond yes throughout three tasks, and half used the left hand). The participants were asked to respond as quickly and accurately as possible. The order of the tasks and the assignment of the left and right keys to yes and no responses were counterbalanced across participants. The participants received 240 stimuli, 120 for each task; 40 objects were shown with a congruent handgrip, 40 with an incongruent handgrip, and 40 with no handgrip (20 yes and 20 no responses, in each case). The stimuli in each task were repeated once in the experiment but were counterbalanced over responses (once on a yes trial and once on a no trial).

The participants received nine practice trials before each task. Each trial in a task began with the presentation of a fixation point for $1,000 \mathrm{msec}$ in the middle of the screen, which was followed by the stimuli (a target object and a word simultaneously) for $700 \mathrm{msec}$. A limited response time (RT) of 3,000 msec was given to the participants. RTs were measured from the onset of the target. The participants were told that the handgrip was irrelevant and that decisions should be based on the object present.

\section{Results}

RTs. RTs were removed if they fell more than three standard deviations from the mean for each participant. 


\section{(A) "Yes" Responses}

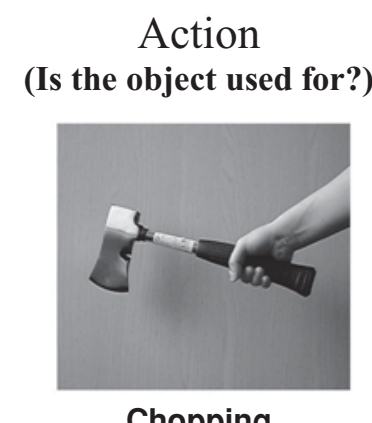

\section{Congruent Handgrip}

\section{Incongruent Handgrip}

No Handgrip

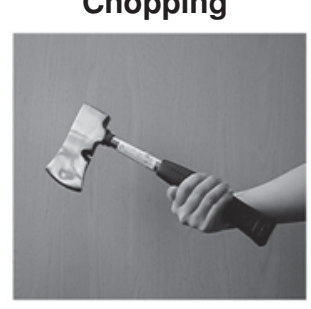

Chopping

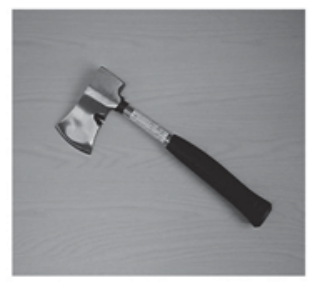

Chopping

\section{Name \\ (Is this the name of the object?)}

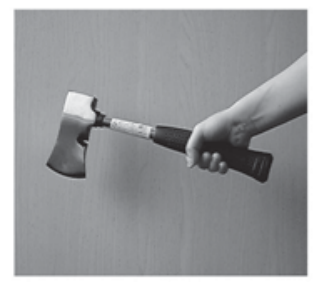

Axe

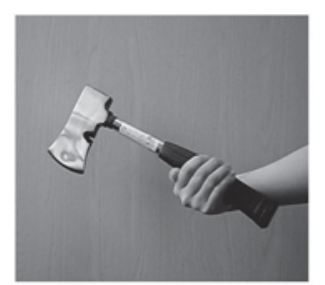

Axe

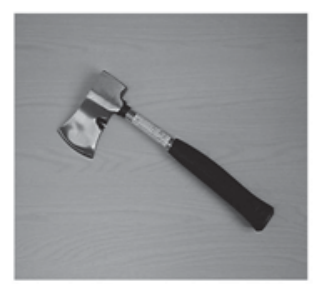

Axe

Figure 2. Example stimuli from Experiment 1. The stimuli are shown for name decision tasks for (A) yes and (B, next page) no trials, as a function of whether the handgrip was congruent, incongruent, or absent (neutral baseline).

The mean RTs were $795 \mathrm{msec}(S E=17.4)$ for object name verification and $845 \mathrm{msec}(S E=20.8)$ for object action verification. The full RT data are shown in Figure 3A. The data were analyzed across participants $\left(F_{1}\right)$ and items $\left(F_{2}\right)$ in repeated measures ANOVAs with three factors: task (name or action verification), response (yes or no), and handgrip (congruent, incongruent, or no handgrip). These revealed significant main effects of task $\left[F_{1}(1,35)=18.2\right.$, $F_{2}(1,59)=107.9$, both $\left.p \mathrm{~s}<.001\right]$, response $\left[F_{1}(1,35)=\right.$ $25.8, F_{2}(1,59)=16.0$, both $p$ s $\left.<.001\right]$, and handgrip $\left[F_{1}(2,70)=3.75, p<.05 ; F_{2}(2,118)=3.0, p s=.054\right]$. A two-way interaction between response and handgrip was borderline significant $\left[F_{1}(2,70)=3.04, p=.054\right.$; $\left.F_{2}(2,118)=4.8, p=.09\right]$. There was also a reliable threeway interaction between task, response, and handgrip $\left[F_{1}(2,70)=4.4, F_{2}(2,118)=4.8\right.$, both $\left.p s<.05\right]$.

The interaction was decomposed by analyzing the effects of the conditions on each task separately. For name verification, there was a significant effect of response $\left[F_{1}(1,35)=12.2, F_{2}(1,59)=8.5\right.$, both $\left.p s<.01\right]$ and a reliable interaction between response and handgrip $\left[F_{1}(2,70)=5.2, p<.01 ; F_{2}(2,118)=3.1, p=.051\right]$. The interaction arose because RTs were longer on yes trials in the incongruent condition, relative to the congruent and no-grip conditions $\left[t_{1}(35)=-2.3, t_{2}(59)=-2.4, p<\right.$ .05 , and $t_{1}(35)=2.25, p<.05, t_{2}(59)=3.0, p<.01$, respectively], whereas the opposite effects occurred on no trials. Taking yes trials alone, there was an effect of handgrip $\left[F_{1}(2,70)=4.5, F_{2}(2,118)=5.0\right.$, both $\left.p s<.05\right]$.

For action verification, there were reliable main effects of response $\left[F_{1}(1,35)=28.3, F_{2}(1,59)=10.8, p<\right.$ $.01]$ and handgrip $\left[F_{1}(1,70)=3.7, p<.05 ; F_{2}(1,118)=\right.$ $2.5, p=.088]$ and again an interaction between response and handgrip $\left[F_{1}(2,70)=2.9, p=.06 ; F_{2}(1,59)=10.8\right.$, $p<.01]$. On yes trials, responses on congruent trials were faster than those on incongruent and no-grip trials $\left[t_{1}(35)=-2.7, p<.05, t_{2}(59)=-1.9, p=.064\right.$, and $t_{1}(35)=-1.9 p=.064, t_{2}(59)=-2.2, p<.05$, respectively]. On no trials, responses on incongruent trials were slower than those on no-grip trials $\left[t_{1}(35)=3.3, t_{2}(59)=\right.$ $2.7, p<.01]$. In order to see whether there was a reliable main effect of congruency, the data were taken for the congruent and incongruent conditions alone. A two-way ANOVA was carried out with grip (congruent or incongruent) and response (yes or no) as factors. There were reliable main effects of grip and response $\left[F_{1}(1,35)=5.3\right.$, 
(B) "No" Responses

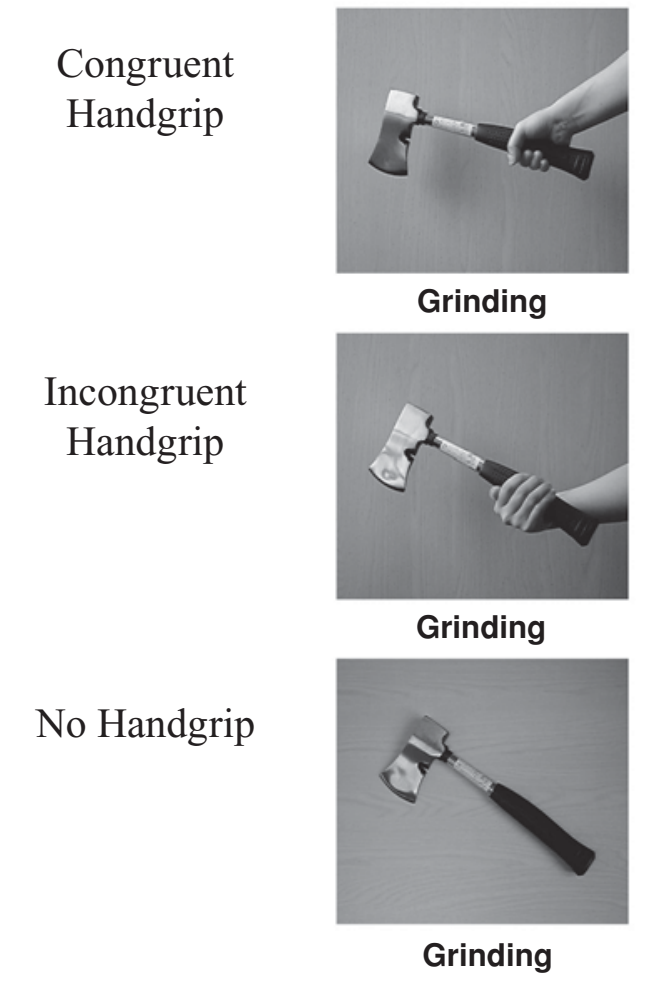

Action
(Is the object used for?)

(Is this the name of the object?)

Figure 2. (Continued).

$p<.05, F_{2}(1,59)=5.7, p<.05$, and $F_{1}(1,35)=29.7$, $p<.001, F_{2}(1,59)=21.7, p<.001$, respectively]. The interaction between grip and response was not significant.

Errors. The means of the error rates for each task were $6.4 \%(S E=0.62 \%)$ for object name judgments and 9.5\% $(S E=0.76 \%)$ for object action judgments. The data are shown in Figure 3B. An overall ANOVA revealed main effects of task $\left[F_{1}(1,35)=19.2, F_{2}(1,59)=17.0\right.$, both $p \mathrm{~s}<.001]$ and response $\left[F_{1}(1,35)=20.0, F_{2}(1,59)=\right.$ 19.2 , both $p \mathrm{~s}<.001]$. There was no interaction between factors.

\section{Discussion}

The action decision task was affected by handgrips to objects. RTs were shorter when the objects were shown with a congruent handgrip than when the objects were depicted with an incongruent handgrip, regardless of the response. For yes trials, RTs also tended to be shorter in the congruent than in the no-grip condition. For the name verification task, there was no overall handgrip effect, although there was some effect on yes trials. These data are consistent with handgrip's having a pervasive effect on action decisions, with any effects being more systematic than those found in name verification. Nevertheless, some effects are apparent for name verification too. If action decisions were contingent on access to the same semantic information as may be required for name retrieval, we would expect the effects of handgrip to be similar across the tasks (for both yes and no responses). The evidence suggests otherwise. This indicates that action decisions are strongly influenced by the visual properties of the environment typically present when objects are used (including the correct handgrip), consistent with there being a direct route from vision to action. It is of interest as well, though, that positive responses in the name verification task also were influenced by handgrip (responses in congruent trials being faster than those in incongruent trials). This in turn suggests that properties associated with action play some role in semantic access and name retrieval too. This fits with data from functional brain imaging, where brain areas associated with action are activated even in object name retrieval tasks (Grabowski, Damasio, \& Damasio, 1998).

\section{EXPERIMENT 2 Hand Movement and Action (Focused Objects)}

In Experiment 2, we examined the effects of hand movements on action and name verification responses to objects. The participants saw objects that were moved in a manner that was either congruent or incongruent with the standard action with the object. The task was to respond to the object and to ignore the movement. In a third, baseline condition, objects were moved in a neutral manner (shifted 

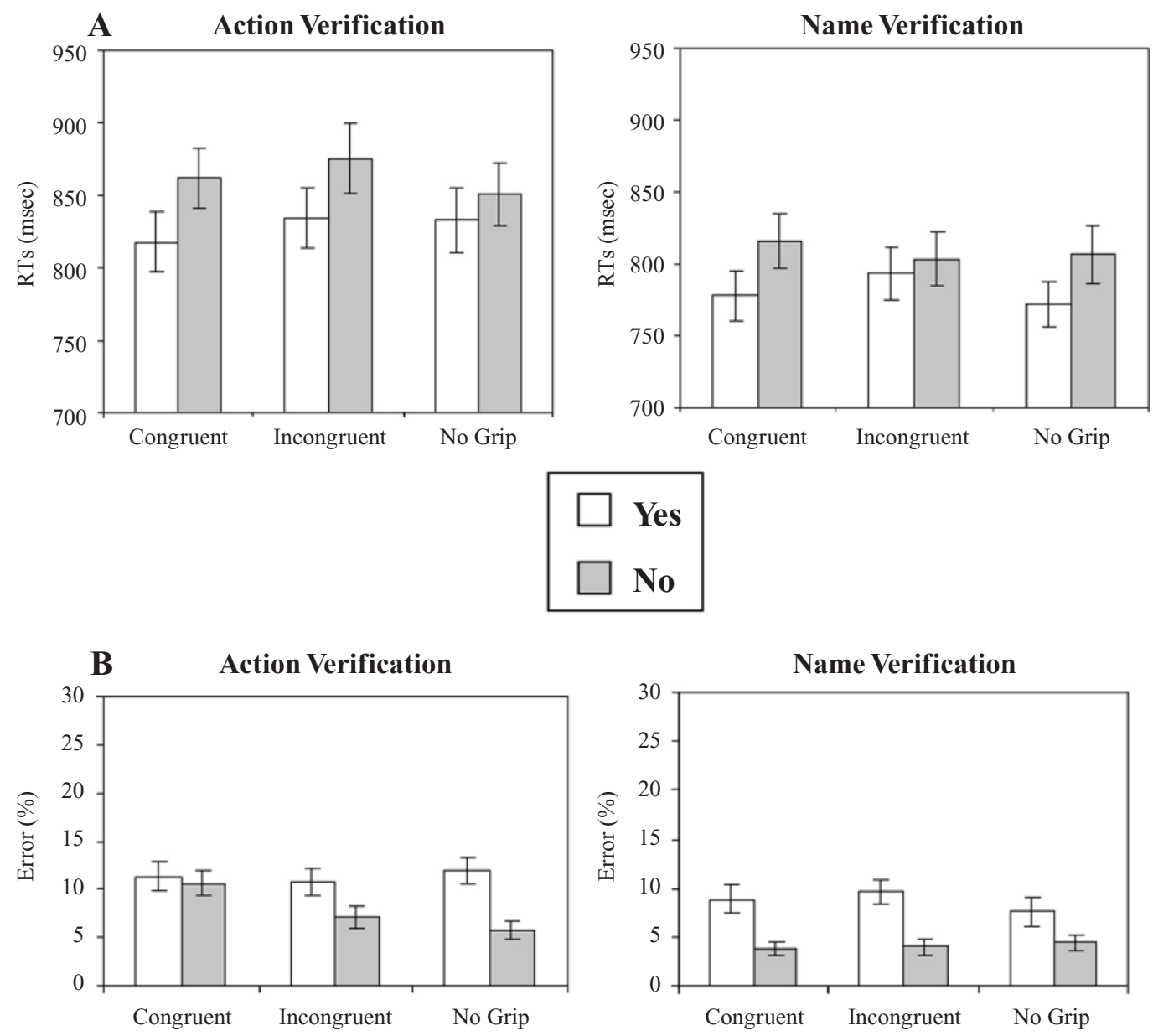

Figure 3. The mean (A) correct response latencies (RTs, with standard errors) and (B) percentages of error (with standard errors) for action and name verification tasks in Experiment 1 (respond to the object and ignore the handgrip).

on the table) that was not associated with a particular category of action. A direct route to action may be influenced by associated motion patterns, whereas object motion may be less important for access to semantic knowledge about objects (required for name verification).

\section{Method}

Participants. There were 24 participants, from 15 to 34 years of age. All the participants had normal or corrected-to-normal vision. All except 4 reported that they were right-handed.

Materials. The target objects were the same as those in Experiment 1. The Appendix shows the action selected for each stimulus. Motion clips of 60 stimuli were taken with a Fujifilm A202 digital camera in three conditions: with a congruent hand movement, with an incongruent hand movement, or with a neutral hand movement (see Figure 4). In the congruent condition, the hand moved the object in a manner consistent with the object's use in everyday life. In the incongruent condition, the hand moved the object in a manner that was incongruent with the object's usual use (e.g., a stirring action for an axe). In the neutral condition, the hand moved the object from right to left. These motion clips were then edited with Adobe Premiere 5.0 in order to match contrast, brightness, and the number of frames to be shown. All the stimuli were compressed so as to show the hand movement within 1,500 msec. Each display consisted of 15 frames, each of which stayed on the screen for $100 \mathrm{msec}$ (in total, 1,500 msec). The frame size of the stimuli was 320 pixels wide $\times 240$ pixels high $\left(7.1^{\circ}\right.$ of visual angle), and the motion clip window was placed at the center of the computer screen throughout the experiment. Simultaneously with the object, a word corresponding to an action or a name was depicted beneath the video clip (as in Experiment 1).

Design and Procedure. The participants had two tasks: name and action verification. The participants were required to decide, by pressing the designated keys, whether the object in the motion clip matched with either the object name or the action label corresponding to the category of action for that object. Each trial in a task began with the presentation of a fixation point for $1,000 \mathrm{msec}$ in the middle of the screen, which was followed by the stimuli (a motion clip and a word) for 1,500 msec. A limited RT of 3,000 msec was given to the participants. RTs were measured from the onset of the target object. The participants were asked to attend to and make their decisions about the objects and to ignore the movements.

\section{Results}

RTs. RTs were omitted if they fell more than three standard deviations from the mean for each participant for 

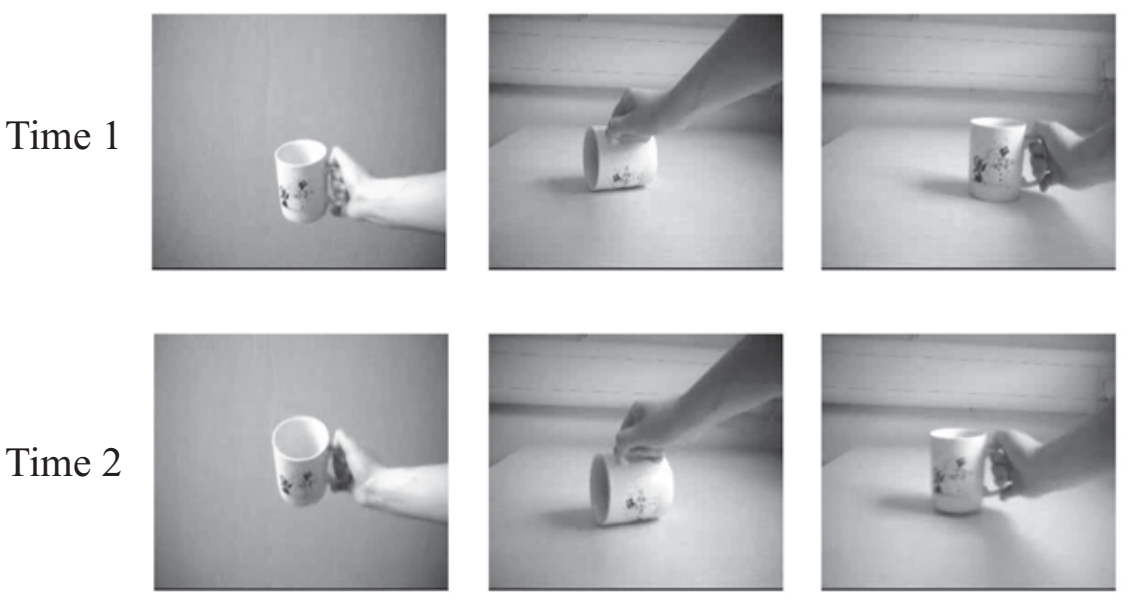

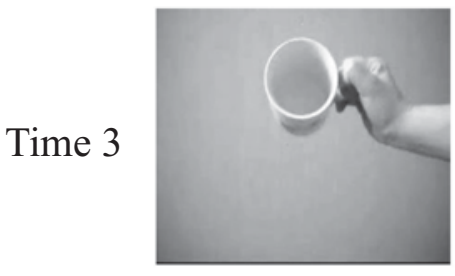

Congruent Hand Movement

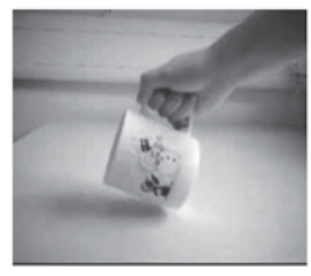

Incongruent

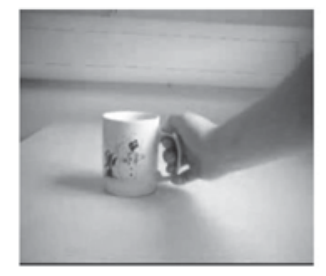

Neutral

\section{Hand Movement Hand Movement}

Figure 4. Example stimuli in Experiment 2. The stimuli are depicted with an action that is either congruent or incongruent with the target object (congruent $=$ the visual action performed with the object; incongruent $=$ an action visually performed with another object). The neutral condition corresponded to the hand's moving the object from right to left on the table. Times 1-3 refer to three consecutive time frames in the video presentation.

each task. The mean RTs were $921 \mathrm{msec}(S E=21.1)$ for name verification and $974 \mathrm{msec}(S E=25.7)$ for action verification. The data were analyzed in a repeated measure ANOVA with three factors: task (name or action verification), response (yes or no), and hand movement (congruent, incongruent, or neutral). There were reliable main effects for each factor [task, $F_{1}(1,23)=11.6, F_{2}(1,23)=$ 13.9 , both $p s<.01$; response, $F_{1}(1,23)=10.6, F_{2}(1,23)=$ 32.3 , both $p \mathrm{~s}<.01$; hand movement, $F_{1}(2,46)=10.4$, $F_{2}(2,46)=8.5$, both $p$ s $\left.<.001\right]$. There was also a reliable two-way interaction between task and hand movement $\left[F_{1}(2,46)=3.9, p<.05\right.$, and $\left.F_{2}(2,46)=3.04, p=.058\right]$. The mean correct RTs are given in Figure $5 \mathrm{~A}$.

As before, the two-way interaction was decomposed by analyzing the data separately for each task. For name verification, there was only an effect of response $\left[F_{1}(1,23)=\right.$ $\left.6.6, p<.05 ; F_{2}(1,59)=8.3, p<.01\right]$. Yes responses were faster than no responses.

For action verification, there were reliable effects of both hand movement $\left[F_{1}(2,46)=12.3, F_{2}(2,118)=8.0\right.$, both $p s<.01]$ and response $\left[F_{1}(1,23)=9.8, F_{2}(1,59)=\right.$ 8.0 , both $p \mathrm{~s}<.01]$. There was no interaction between hand movement and response. RTs were shorter when hand movements were congruent, relative to incongruent $\left[t_{1}(23)=-4.38, t_{2}(59)=-3.64\right.$, both $\left.p \mathrm{~s}<.01\right]$, and also when hand movements were neutral, relative to incongruent $\left[t_{1}(23)=-5.0, t_{2}(59)=-3.29\right.$, both $p \mathrm{~s}<$ .01]. There was no difference in RTs between congruent and neutral hand movements $\left[t_{1}(23)=-0.15, t_{2}(59)=\right.$ -0.31 , both $p \mathrm{~s}>.05]$.

Errors. The means of the error rates for each task were $3.2 \%(S E=0.49)$ for name verification and $5.2 \%$ $(S E=0.61)$ for action verification. The percentages of errors are illustrated in Figure 5B. The repeated measure ANOVA revealed that there were reliable effects of task $\left[F_{1}(1,23)=13.9, F_{2}(1,59)=8.6\right.$, both $p$ s $\left.<.01\right]$, response $\left[F_{1}(1,23)=32.3, F_{2}(1,59)=19.3\right.$, both $p \mathrm{~s}<$ $.001]$, and hand movement $\left[F_{1}(2,46)=8.5, p<.01\right.$, and $\left.F_{2}(2,118)=4.2, p \mathrm{~s}<.05\right]$. There were also interactions between task and response $\left[F_{1}(1,23)=6.4, p<\right.$ $\left..05 ; F_{2}(1,59)=3.6, p=.064\right]$ and between response and hand movement $\left[F_{1}(2,46)=6.6, F_{2}(2,118)=5.3\right.$, both $p \mathrm{~s}<.01]$. The interaction between task and hand movement was borderline significant, especially for the analysis across participants $\left[F_{1}(2,46)=3.04, p=.058\right.$; $\left.F_{2}(2,118)=2.5, p=.09\right]$. For name and action verification, more errors were made on yes than on no responses. In particular, on yes trials for action verification, significantly fewer errors occurred when congruent and neutral hand movements were shown to viewers, relative to 

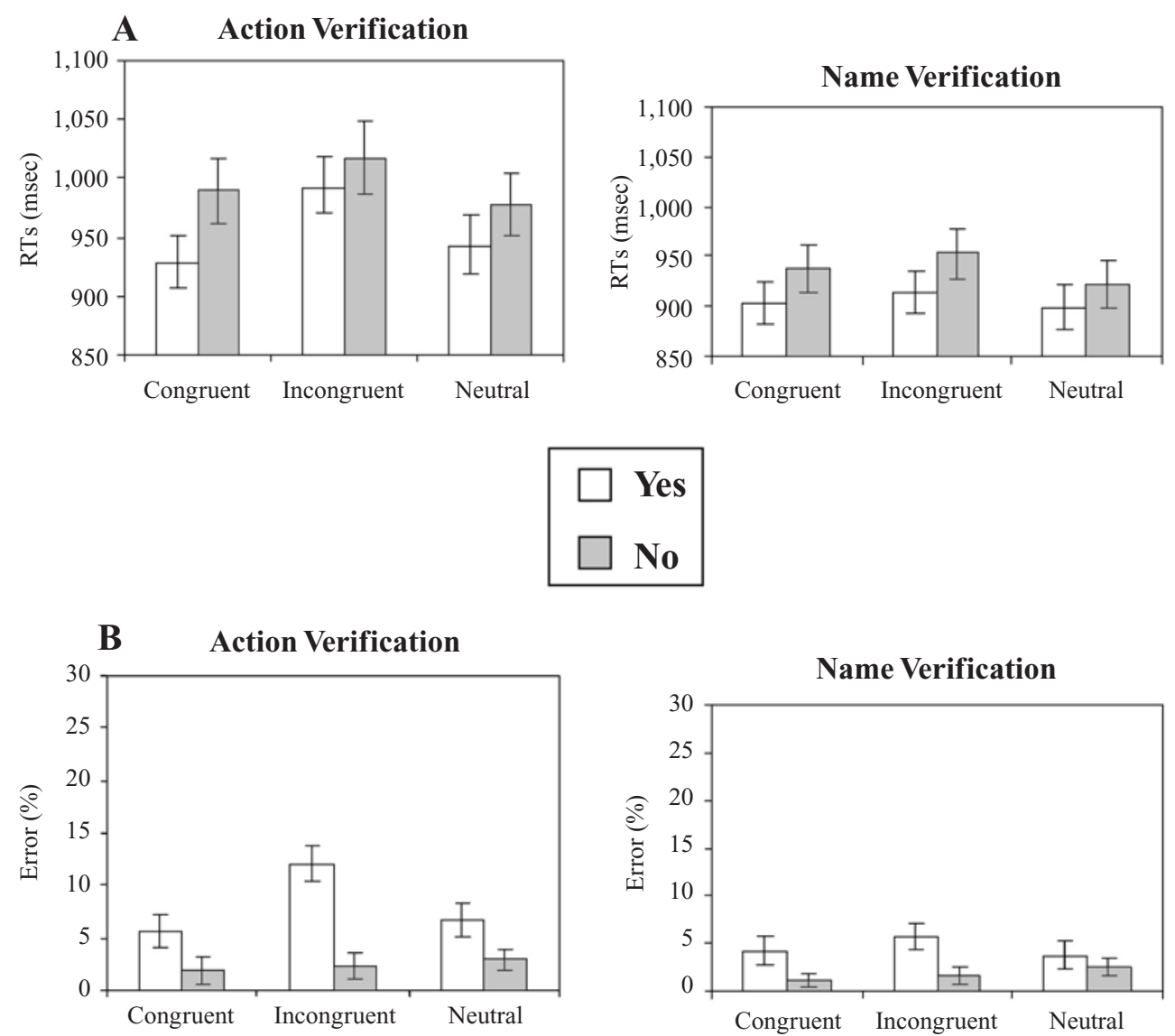

Figure 5. The mean (A) correct response latencies (RTs, with standard errors) and (B) percentages of errors (with standard errors) for action and name verification tasks in Experiment 2 (respond to the object and ignore the hand movement).

when incongruent hand movements were shown $\left[t_{1}(23)=\right.$ $-3.7, t_{2}(59)=-2.9$, both $p \mathrm{~s}<.01$, and $t_{1}(23)=3.3$, $t_{2}(59)=2.6$, both $p \mathrm{~s}<.01$, respectively].

\section{Discussion}

Action verification was strongly affected by the type of hand movement. Relative to the neutral baseline, incongruent hand movements slowed RTs, whereas congruent hand movements tended to facilitate performance, and there was no interaction of this effect with response. For name verification, there was no effect of hand movement. Thus, it cannot be argued that the movements affected performance simply because they were more salient than the objects (and see Experiment 3, where we examined the reverse task, with responses made to the movements rather than to the objects). For name verification, object movements could be effectively ignored.

\section{EXPERIMENT 3}

\section{Respond to Hand Movement}

In Experiment 2, the participants responded to target objects, and they were asked to ignore hand movements. In Experiment 3, we reversed the task so that the partici- pants made responses to the movement and were asked to ignore the object. In name verification, the participants had to verify whether the hand in the motion clips would be using the object named below, rather than the object being held in the hand. The action verification task was to decide whether the hand action performed in the clips matched with the action name, regardless of the object presented. For name verification, we may now expect a different pattern of results, as compared with Experiment 2 (in which the type of hand movement had no effect). Name verification may tend to stress the object associated with the task, since object names refer to objects more usually than to types of motion. In this case, the congruency of the object may influence the time taken to match the hand motion to the object name. For action verification, we propose that the object being used is intimately linked with the type of motion that characterizes the action. From this, we may again expect that the congruency of the object will affect action decision times to the hand movements.

\section{Method}

Participants. There were 12 participants from 19 to 24 years of age. All the participants had normal or corrected-to-normal vision. All the participants reported that they were right-handed. 
Materials. The target objects were the same as those in Experiment 2 . The motion clips for 60 stimuli were the same as those in Experiment 2, except for the neutral condition. The neutral condition in Experiment 2 was replaced by a condition in which the hand movement was made without an object. The hand movement in this neutral condition matched the movement used in the congruent and incongruent conditions. Without objects, clips of pantomimed hand movements were taken with a Fujifilm A202 digital camera. Even though the words were randomly assigned to the stimuli for the no trials, some possibly confusing words were avoided for matching the object targets. For example, pounding for a meat hammer was not paired with hitting for a hammer, and wiping for a $j$ cloth was not paired with sweeping for a brush or painting for a paintbrush, and so on. This was done to avoid confusion, since some actions may be labeled in several ways.

Design and Procedure. The participants had two tasks: name and action verification. The participants received two conditions. In the name condition, they were required to decide, by pressing the designated keys, whether the hand movement made by the actor in the video matched the name presented (e.g., a drinking action being presented along with the name cup). In the action condition, they decided whether the hand movement matched the action label provided (drinking to a drinking action). The details of the design and procedure for Experiment 3 were otherwise the same as those in Experiment 2.

\section{Results}

RTs. RTs were omitted if they fell more than three standard deviations from the mean for each participant for each task. The mean RTs in two tasks were $1,355 \mathrm{msec}$ $(S E=51.4)$ for name verification and $1,298 \mathrm{msec}(S E=$ 46.1) for action verification. It is interesting that this pattern is the reverse of that for the task effects in Experiments 1 and 2, suggesting that hand movements are more usually classified for type of action than for object identification. The RT data were analyzed in a repeated measure ANOVA with three factors: task (name or action), response (yes or no), and hand movement (congruent, incongruent, or hand movement alone). There were strongly significant effects of response $\left[F_{1}(1,11)=7.0\right.$, $p<.05$, and $\left.F_{2}(1,47)=2.9, p \mathrm{~s}=.096\right]$ and hand movement $\left[F_{1}(2,22)=103.1, F_{2}(2,94)=27.6\right.$, both $\left.p s<.001\right]$ and an interaction between response and hand movement $\left[F_{1}(2,22)=6.6, F_{2}(2,94)=5.2\right.$, both $\left.p \mathrm{~s}<.01\right]$. The mean correct RTs and percentages of errors are illustrated in Figures 6A and 6B.

The two-way interaction was separately analyzed for each response. There was no effect of task. For both yes and no responses, there were significant effects of hand movement $\left[F_{1}(2,22)=48.9, F_{2}(2,104)=22.9\right.$, both $p$ s $<$ .001 , for yes responses; $F_{1}(2,22)=35.7, F_{2}(2,106)=$ 15.7 , both $p s<.001$, for no responses]. Verification for both names and actions was affected by the congruency of the hand movement. A paired $t$ test for yes responses in name decisions revealed that there were significant differences between each condition $\left[t_{1}(11)=-6.9, t_{2}(58)=\right.$ -5.4 , both $p \mathrm{~s}<.001$, for congruent vs. incongruent hand movements; $t_{1}(11)=-6.7, t_{2}(58)=-3.0$, both $p s<.01$, for congruent vs. movement alone; $t_{1}(11)=3.9, t_{2}(57)=$ 2.7 , both $p s<.01$, for incongruent vs. hand movement alone]. The same generally held for the no responses in name decisions $\left[t_{1}(11)=-5.9, t_{2}(58)=-3.6\right.$, both $p \mathrm{~s}<$ .001 , for congruent vs. incongruent hand movements; $t_{1}(11)=-2.5, p<.05$, and $t_{2}(56)=-1.5, p=.14$, for congruent vs. movement alone; $t_{1}(11)=3.1, t_{2}(55)=$ 2.9 , both $p \mathrm{~s}<.01$, for incongruent vs. movement alone]. A paired $t$ test for yes responses was applied for action decisions. This revealed that there were significant differences between each condition $\left[t_{1}(11)=-6.2, t_{2}(54)=\right.$ -5.5 , both $p \mathrm{~s}<.001$, for congruent vs. incongruent hand movement; $t_{1}(11)=-4.5, t_{2}(58)=-4.9$, both $p s<.001$, for congruent vs. movement alone; $t_{1}(11)=1.7, p=.1$, and $t_{2}(53)=0.8, p=.4$, for incongruent vs. movement alone]. The same generally held for the no responses in action decisions $\left[t_{1}(11)=-6.6, p<.001\right.$, and $t_{2}(58)=$ $-2.4, p<.05$, for congruent vs. incongruent hand movements; $t_{1}(11)=-3.0, t_{2}(56)=-3.0$, both $p \mathrm{~s}<.05$, for congruent vs. movement alone; however $t_{1}(11)=0.68$, $t_{2}(55)=0.15$, both $p s>.5$, for incongruent vs. movement alone].

Errors. The mean of error rates for each task were $15.6 \%(S E=1.55 \%)$ and $15.6 \%(S E=1.30 \%)$ for name and action verifications, respectively. A repeated measure ANOVA showed that there was a significant effect of hand movement $\left[F_{1}(2,22)=32.5, F_{2}(2,118)=21.4\right.$, both $p \mathrm{~s}<.001]$ and an interaction between response and hand movement $\left[F_{1}(2,22)=9.9, p<.001 ; F_{2}(2,118)=3.8\right.$, $p<.05]$. For the positive responses, there was a reliable effect of hand movement $\left[F_{1}(2,22)=29.4, F_{2}(2,118)=\right.$ 21.6 , both $p \mathrm{~s}<.001]$, and the same held for negative responses $\left[F_{1}(2,22)=18.3, F_{2}(2,118)=6.4\right.$, both $p$ s $<$ $.01]$. A paired $t$ test for the positive responses in name verification revealed there were significant differences between the conditions $\left[t_{1}(11)=-5.4, t_{2}(59)=-6.0\right.$, both $p \mathrm{~s}<.001$, for congruent vs. incongruent hand movements; $t_{1}(11)=-3.5, t_{2}(59)=-4.0$, both $p \mathrm{~s}<.01$, for congruent vs. movement alone; $t_{1}(11)=1.9, p=.09$, and $t_{2}(59)=1.2, p=.24$, for incongruent vs. hand movement alone]. A paired $t$ test for the negative responses in name verification showed that there were significant differences between congruent and incongruent hand movements $\left[t_{1}(11)=-4.2, t_{2}(59)=-2.7\right.$, both $p \mathrm{~s}<$ $.01]$ and between congruent movements and movements alone $\left[t_{1}(11)=-6.2, t_{2}(59)=-3.6 p<.01\right]$, but not between incongruent movements and movements alone $\left[t_{1}(11)=-1.1, p=.305 ; t_{2}(59)=-0.77, p=.447\right] . \mathrm{A}$ paired $t$ test for yes responses in action decisions showed there were significant differences between each condition $\left[t_{1}(11)=-4.7, t_{2}(59)=-4.7\right.$, both $p \mathrm{~s}<.001$, for congruent vs. incongruent hand movement; $t_{1}(11)=-5.9$, $t_{2}(59)=-5.0$, both $p \mathrm{~s}<.001$, for congruent movement vs. movement alone; however, $t_{1}(11)=1.3, p=.22$, and $t_{2}(59)=1.1, p=.29$, for incongruent movement vs. movement alone]. The same generally held for the no responses in action decisions $\left[t_{1}(11)=-4.0, t_{2}(59)=\right.$ -2.8 , both $p \mathrm{~s}<.01$, for congruent vs. incongruent hand movements; $t_{1}(11)=-3.4, p<.01$, and $t_{2}(59)=-2.4$, 

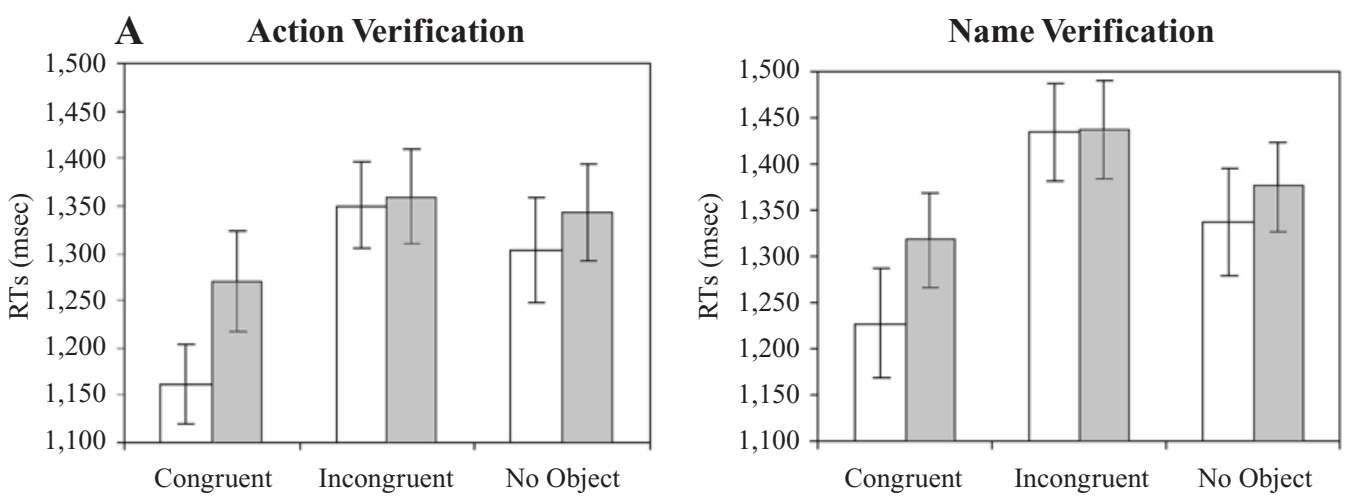

Yes

No

B

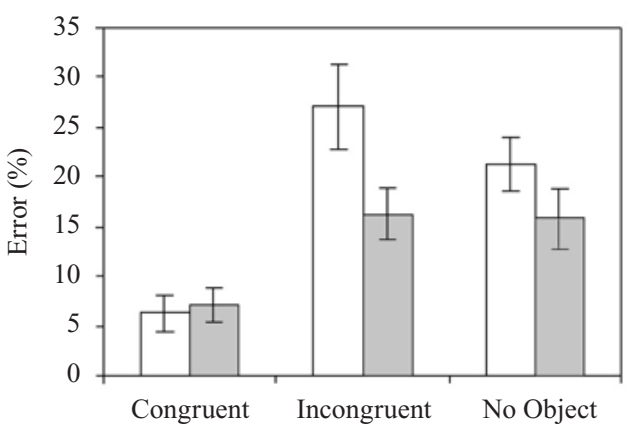

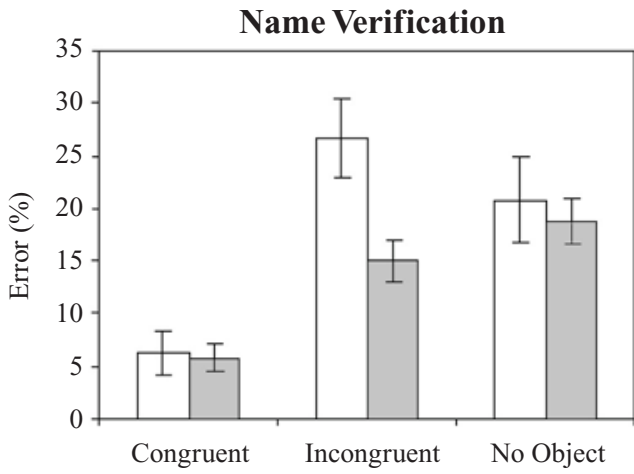

Figure 6. The mean (A) correct response latencies (RTs, with standard errors) and (B) percentages of errors (with standard errors) for action and name verification tasks in Experiment 3 (respond to the hand movement and ignore the object).

$p<.05$, for congruent movement vs. movement alone; however, $t_{1}(11)=0.11, t_{2}(59)=0.09$, both $p \mathrm{~s}>.5$, for incongruent movement vs. movement alone].

\section{Discussion}

In this experiment, both name and action judgments were affected by the congruency between the action being performed and the object being used. In both tasks, RTs were shorter and error rates reduced if the object was congruent with the action than if it was incongruent, and performance in the no-object (hand movement alone) condition tended to fall in between. The results are of interest for several reasons. One is that, given the tendency to classify hand movements in terms of action (verified by the overall RT advantage for action decisions here), there might be little "room" for the object being used to affect performance. The data refute this. There was a strong effect of object congruency. This is consistent with action information associated with the objects being made available rapidly and in a relatively automatic fashion, influencing performance even when its effect was disruptive (e.g., on incongruent trials). Second, the effect of object congruency on name verification here, and the contrasting lack of an effect of movement congruency in Experiment 2, fits with the proposal that name verification is linked to object, rather than to movement, processing. This linkage produces asymmetrical effects on performance. Third, the data are interesting given the existence of distinct neural regions specializing in motion and in form perception (see Buccino et al., 2001; Chao, Haxby, \& Martin, 1999). Studies of neuroimaging further suggest that participants may selectively activate featurespecific regions of the cortex when asked to attend to independent properties of objects (e.g., form or motion; see Saenz, Buracas, \& Boynton, 2002). Hence, we might expect minimal influence on attention of stimuli defined by one set of features (the form of an object) and responses to another (type of object motion). This was clearly not the case here (nor was it the case when action decisions were made in Experiment 2). At least for complex patterns of motion, which must be classified on the basis of stored knowledge, the properties of objects being held cannot be 
ignored. This result matches those of prior work showing semantic priming from object form even when participants were asked to attend and respond to object motion (Boucart, Humphreys, \& Lorenceau, 1995). It appears that form information cannot be ignored in such tasks. A final point is that RTs were longer here than in Experiments 1 and 2, for both name and action verification. This likely occurred because in Experiment 3, the participants needed to integrate the images over time in order to respond to the type of motion, whereas they could respond directly to the object in Experiments 1 and 2.

\section{GENERAL DISCUSSION}

The data from the present experiments show effects of handgrip and hand movement on name and action verification for objects, along with effects of the object being held on judgments about the type of hand movement performed. In Experiments 1 and 2, the participants made responses to the object present on each trial. Experiment 1 demonstrated an effect of handgrip, which was present for both action and name verification tasks (although it held only across both yes and no responses for action decisions). Experiment 2 revealed an effect of the type of hand movement performed, but this held only for action decisions. Experiment 3 showed effects of object congruency on both action and name decisions to hand movements.

The effect of handgrip on yes responses in name verification in Experiment 1 fits with data from functional imaging studies suggesting that action information linked to objects is retrieved even when only object identification is required (Chao \& Martin, 2000; Grèzes \& Decety, 2002; Grèzes, Tucker, Ellis, \& Passingham, 2003). From the imaging data alone, though, it is difficult to know whether the activation of action-based regions plays any functional role in object identification. The present data indicate that at least some kinds of action information do (handgrip, but not type of hand movement, in Experiment 2). Nevertheless, the effects of action-associated information (handgrip and type of movement) tended to be consistent across decisions (affecting both yes and no responses) and stronger when action, rather than name, decisions were made to the objects (Experiments 1 and 2 ). This is in accordance with the predictions of a dualroute account of object processing, in which action-based representations are activated by both the structural and the semantic properties of objects (Riddoch et al., 1989; Yoon et al., 2002). According to this account, action decisions will be sensitive to the visual properties of episodes of action performed with objects, including handgrip and movement, because (1) these associated properties of action are represented in our stored knowledge of the actions performed with objects and (2) action knowledge is evoked directly by objects, without depending on prior access to more abstract semantic knowledge (e.g., knowledge about where objects might typically be found). In contrast, name decisions depend on access to semantic knowledge, which is abstracted from the episodic prop- erties associated with object use-for example, how a particular object is gripped or moved. As a consequence, name verification should be less affected by these associated, action-related properties.

Although there were asymmetric effects of handgrip and (particularly) type of movement on action and name judgments to objects, we found effects of equivalent consistency and magnitude when the same judgments were applied to hand movements (Experiment 3). The effects on action decisions fit with the proposal that object and action-associated visual information (e.g., type of movement) were intimately linked in action judgments, providing reciprocal effects. The pattern of performance for name verification, though, is consistent with name verification being biased toward objects, rather than toward movement patterns. Hence, the object influences name verification of the action (Experiment 3), but not vice versa (Experiment 2). This fits with name retrieval being semantically mediated (see Figure 1), when it operates both with respect to objects and with respect to hand movements.

The results are less easy to account for in theories that do not distinguish between semantic and action knowledge. For example, consider an alternative account in which there is a single knowledge store that incorporates verbal, visual, and motor/action components, depending on the situation that produced the trace. ${ }^{3}$ Furthermore, let retrieval from such a system operate along the lines of Hintzman's $(1984,1986)$ MINERVA model, in which what is retrieved is a weighted average of all activated traces, where the weighting depends on the similarity of the stimulus and the trace. The episodic properties of an object are frequently paired with the object name, so that many traces are activated in the object name condition across different episodic contexts. Under these circumstances, variations in the grip or in the movement pattern may matter little. In contrast, the appearance of the object and a verb describing its action may be a less frequent event, dependent on the activation of a specific instance of object use. In this case, variations in the grip and the action may be important. Now, for this view, the overall RT for the task may be taken as a measure of the number of episodes activated during retrieval. Name verification is faster than action verification for objects, reflecting the greater number of traces activated by the conjunction of the object and the name. Taking the overall RTs for judgments made to hand movements (Experiment 3), we would then make the opposite prediction. RTs are shorter for action than for name verification, presumably because the action and the action name appear more often together than the action and the object's name. We would then expect congruency effects to be larger for name verification than for action verification. This was not the case. Furthermore, this single-route account provides no ready explanation for the double dissociations found in the neuropsychological literature (e.g., between optic aphasia and semantic dementia, on the one hand, and visual apraxia, on the other). In contrast to this, the dual-route account 
can accommodate the neuropsychological data, and it also accounts for the symmetrical effects of object congruency on action and name verification (since in both cases, performance is contingent on action knowledge).

\section{Congruency Effects}

There are numerous demonstrations in the literature showing that responses are faster when irrelevant stimulus information is congruent with relevant stimulus information, with performance also being slowed when the relevant and the irrelevant information are incongruent; the Stroop effect (Stroop, 1935; see MacLeod, 1991, 1992), the Simon effect (Simon \& Small, 1969) and picture-word interference effects (Glaser, 1992) are three of these. The present results can be added to this list. As in these other studies, the disruptive effects of incongruent information tended to be stronger than the beneficial effects of congruent information. In terms of the Stroop effect, Cohen, Dunbar, and McClelland (1990) have modeled this in a connectionist system, in which activation increases as a sigmoid function. Due to this function, there is a limit on how much performance can be facilitated by congruent stimulus information, relative to a neutral baseline, as the activation reaches an asymptote. On the other hand, incongruent information exerts a larger effect, because it can shift activation into a linear part of the function, where greater performance changes accrue for a given change in the input. A similar account can be put forward to explain the stronger effects on incongruent than on congruent trials here.

In terms of congruency effects, the results from Experiments 2 and 3 are particularly interesting, since, to the best of our knowledge, they are the first to demonstrate effects of congruency between object properties and the type of movement being performed. As we have noted, there is good evidence to suggest that movement information is processed independently of information about object identity. For example, distinct brain regions seem to have been activated for movement and object identity in studies in which functional imaging has been used (e.g., Buccino et al., 2001; Chao et al., 1999; Grafton et al., 1996), and neuropsychological patients can have selective loss of object identity but not of movement (e.g., Riddoch \& Humphreys, 1987) or of movement but not of object identification (see Heywood \& Zihl, 1999). Given this, one might imagine that observers ought to be able to attend selectively to each type of information, minimizing effects of congruency from the irrelevant stimulus. This was not the case. Instead, it appears that information about object movement is retrieved when we make action decisions to objects, whereas information about the object involved is coded when we make action and name judgments to hand movements. This fits with brain-imaging data showing that the class of object used here (tools) activates motion areas even under static viewing conditions and in object identification tasks (e.g., Buccino et al., 2001; Chao \& Martin, 2000; Decety et al., 1994; Grafton et al., 1996; Grèzes \& Decety, 2002; Kellenbach, Brett, \& Patterson,
2003; Kourtzi \& Kanwisher, 2000). Also, visual attention can be led by an implicit recognition of actions with graspable objects when the objects are presented to viewers (Handy, Grafton, Shroff, Ketay, \& Gazzaniga, 2003). The data suggest that although there are separate brain regions involved in processing the different types of information, the activation derived is interactive and jointly influential. It would clearly be of interest to assess whether reciprocal effects occur when hand actions are viewed, so that brain regions associated with the retrieval of object identity are activated.

\section{A Direct Route to Action, Simulation, and Affordances}

The direct route account that we have proposed fits with other converging evidence both from normal observers (see Craighero et al., 1998, 1999; Tucker \& Ellis, 1998) and from neuropsychological patients (see, e.g., Hillis \& Caramazza, 1995; Riddoch \& Humphreys, 1987; Riddoch et al., 1989). The account holds that there exist routines that associate actions with the structural properties of objects (the direct route), along with those that associate structural properties of objects with abstracted semantic knowledge (the indirect route). Other authors, however, have taken an even more radical view, suggesting that all conceptual knowledge is grounded in perceptual representations and that sensory-motor associations are called upon for conceptual reasoning, even with purely linguistic stimuli (Barsalou, 1999). There is, indeed, evidence that sensory motor actions moderate linguistic judgments. For example, Glenberg and Kaschak (2002) had participants judge whether sentences were sensible by making a response requiring a movement either toward or away from the body. When a sentence implied an action in one direction, responses were difficult to make if they went in the opposite direction. These data are consistent with sensory-motor representations being recruited even in tasks that could, in theory, be performed purely in the language domain. However, the differential effects that we have reported (particularly the effects of object motion in Experiment 2) indicate that action and name decisions are dependent to different degrees on visual properties of action associated with when the objects are used (action decisions being influenced to a greater extent). In other studies (Yoon \& Humphreys, 2005), we have found opposite results, with semantic priming effects being larger on name than on action decisions. These opposite effects are consistent with some separate processes being involved in the tasks, rather than there being common sensory-motor representations (cf. Barsalou, 1999). Nevertheless, the existence of direct sensory-motor associations for objects is necessary if such associations are to be recruited for other cognitive tasks (including language reasoning). Furthermore, according to a sensory-motor view of cognition, a variety of cognitive activities depend on simulation, where particular sensory-motor associations are reactivated on the basis of the constraints of the participant's goal (Barsalou, 1999). These associations 
can involve links between whole objects and actions, as well as links between part representations (individual features of objects, subcomponents of an action). The notion that the activation of sensory-motor knowledge is contingent on the participant's goals fits with the idea of an affordance, as proposed by Gibson (1979). Here, the goal of an observer will shape which features are weighted in an object representation and which components of action are activated in turn. Riddoch et al. (1998) have provided neuropsychological evidence for this. They reported data from a patient who found it difficult to prevent an afforded action being made to an object when the action overlapped with some of the task rules (e.g., to grasp the object with a particular hand). However, these afforded actions decreased when they were not part of the task set (e.g., when the task required pointing rather than grasp responses). On our view, object classification should be viewed as a flexible process dependent on an intersection between the task set, sensory-motor associations, and abstracted semantic features of objects. The direct pathway that we have proposed, linking object representations to action, is a necessary component in such a flexible categorization system.

\section{REFERENCES}

Barsalou, L. W. (1999). Perceptual symbol systems. Behavioral \& Brain Sciences, 22, 577-660.

Beauvois, M. F. (1982). Optic aphasia: A process of interaction between vision and language. Philosophical Transactions of the Royal Society of London: Series B, 298, 35-47.

Biederman, I., \& CoOper, E. E. (1991). Evidence for complete translational and reflectional invariance in visual object priming. Perception, 20, 585-593.

Boucart, M., Humphreys, G. W., \& Lorenceau, J. (1995). Automatic access to object identity: Attention to global information, not to particular physical dimensions, is important. Journal of Experimental Psychology: Human Perception \& Performance, 21, 584-601.

Buccino, G., Binkofski, F., Fink, G. R., Fadiga, L., Fogassi, L., GalLESE, R. J., ET AL. (2001). Action observation activates premotor and parietal areas in a somatotopic manner: An fMRI study. European Journal of Neuroscience, 13, 400-404.

Chainay, H., \& Humphreys, G. W. (2002). Privileged access to action for objects relative to words. Psychonomic Bulletin \& Review, 9, 348-355.

ChaO, L. L., Haxby, J. V., \& Martin, A. (1999). Attribute-based neural substrates in temporal cortex for perceiving and knowing about objects. Nature Neuroscience, 2, 913-919.

ChaO, L. L., \& Martin, A. (2000). Representation of manipulable manmade objects in the dorsal stream. NeuroImage, 12, 478-484.

Cohen, J. D., Dunbar, K., \& McClelland, J. L. (1990). On the control of automatic processes: A parallel distributed processing account of the Stroop effect. Psychological Review, 97, 332-361.

Coslett, H. M., \& SafFran, E. M. (1992). Optic aphasia and the right hemisphere: A replication and extension. Brain \& Language, 13, 9598.

Craighero, L., Fadifa, L., Rizzolatti, G., \& Umiltà, C. (1998). Visuomotor priming. Visual Cognition, 5, 109-126.

Craighero, L., Fadifa, L., Rizzolatti, G., \& Umiltà, C. (1999). Action for perception: A motor-visual attentional effect. Journal of Experimental Psychology: Human Perception \& Performance, 25, 1673-1692.

Decety, J., Perani, D., Jeannerod, M., Bettinardi, V., Woods, R., Mazziotta, J. C., \& FAZIO, F. (1994). Mapping motor representations with positron emission tomography. Nature, 371, 600-602.
DeRenzi, E., Faglioni, P., \& Sorgato, P. (1982). Modality-specific and supramodal mechanisms of apraxia. Brain, 105, 301-312.

Devlin, J. T., Moore, C. J., Mummery, C. J., Gorno-Tempini, M. L., Phillips, J. A., NopPeneY, U., ET AL. (2002). Anatomic constraints on cognitive theories of category specificity. NeuroImage, 15, 675-685.

Gibson, J. J. (1979). The ecological approach to visual perception. Boston: Houghton Mifflin.

Glaser, W. R. (1992). Picture naming. Cognition, 42, 61-105.

Glenberg, A. M., \& KaschaK, M. P. (2002). Grounding language in action. Psychonomic Bulletin \& Review, 9, 558-565.

Grabowski, T., Damasio, H., \& Damasio, A. R. (1998). Premotor and prefrontal correlates of category-related lexical retrieval. NeuroImage, 7, 232-243.

Grafton, S. T., Arbib, M. A., Fadiga, L., \& Rizzolatti, G. (1996). Localization of grasp representations in humans by positron emission tomography: 2. Observation compared with imagination. Experimental Brain Research, 112, 103-111.

GRÈzes, J., \& DeCETy, J. (2002). Does visual perception of object afford action? Evidence from a neuroimaging study. Neuropsychologia, 40, 212-222.

Grèzes, J., Tucker, M., Ellis, R., \& Passingham, R. E. (2003). Objects automatically potentiate action: An fMRI study of implicit processing. European Journal of Neuroscience, 17, 2735-2740.

Handy, T. C., Grafton, S. T., Shroff, N. M., Ketay, S., \& GazzaNIGA, M. S. (2003). Graspable objects grab attention when the potential for action is recognized. Nature Neuroscience, 6, 421-427.

HeYwood, C. A., \& ZiHL, J. (1999). Motion blindness. In G. W. Humphreys (Ed.), Case studies in the neuropsychology of vision (p. 1-16). Hove, U.K. Psychology Press.

Hillis, A., \& Caramazza, A. (1995). Cognitive and neural mechanisms underlying visual and semantic processing: Implication from "optic aphasia." Journal of Cognitive Neuroscience, 7, 457-478.

HinTZMAN, D. L. (1984). MINERVA 2: A simulation model of human memory. Behavior Research Methods, Instruments, \& Computers, 16, 96-101.

Hintzman, D. L. (1986). "Schema abstraction" in a multiple-trace memory model. Psychological Review, 93, 411-428.

Hodges, J. R., Bozeat, S., Lambon Ralph, M. A., Patterson, K., \& SpatT, J. (2000). The role of conceptual knowledge in object use: Evidence from semantic dementia. Brain, 123, 1913-1925.

Hodges, J. R., Spatt, J., \& Patterson, K. (1999). "What" and "how": Evidence for the dissociation of object knowledge and mechanical problem-solving skills in the human brain. Proceedings of the $\mathrm{Na}$ tional Academy of Sciences, 96, 9444-9448.

Humphreys, G. W. (2001). Objects, affordances . . . action! The Psychologist, 14, 408-412.

Humphreys, G. W., \& RidDoch, M. J. (2001). Detection by action: Neuropsychological evidence for action-defined templates in search. Nature Neuroscience, 4, 84-88.

Humphreys, G. W., \& RidDOCH, M. J. (2003). From vision to action, and action to vision: A convergent route approach to vision, action and attention. In D. Irwin \& B. Ross (Eds.), Cognitive vision: Psychology of learning and motivation (Vol. 42, pp. 225-264). New York: Elsevier.

Jeannerod, M., Decety, J., \& Michel, F. (1994). Impairment of grasping movements following a bilateral posterior parietal lesion. Neuropsychologia, 32, 369-380.

Kellenbach, M. L., Brett, M., \& Patterson, K. (2003). Actions speak louder than functions: The importance of manipulability and action in tool representation. Journal of Cognitive Neuroscience, 15, 30-46.

KourtzI, Z., \& Kanwisher, N. (2000). Activation in human MT/MST by static images with implied motion. Journal of Cognitive Neuroscience, 12, 48-55.

Lhermitte, F., \& Beauvois, M. F. (1973). A visual-speech disconnection syndrome: Report of a case with optic aphasia. Brain, 96, 695-714.

MACLEOD, C. M. (1991). Half century of research on the Stroop effect: An integrative review. Psychological Bulletin, 109, 163-203.

MacLeod, C. M. (1992). The Stroop task: The "gold standard" of attentional measures. Journal of Experimental Psychology: General, 121, 12-14.

Manning, L., \& CAMPBell, R. (1992). Optic aphasia with spared ac- 
tion naming: A description and possible loci of impairment. Neuropsychologia, 30, 587-592.

Ochipa, C., Rothi, L. J., \& Heilman, K. M. (1992). Conceptual apraxia in Alzheimer's disease. Brain, 115, 1061-1071.

Pilgrim, E., \& HumphreYs, G. W. (1991). Impairment of action to visual objects in a case of ideomotor apraxia. Cognitive Neuropsychology, 8, 459-473.

RidDOCh, M. J., Edwards, M. G., Humphreys, G. W., West, R., \& Heafield, T. (1998). An experimental study of anarchic hand syndrome: Evidence that visual affordances direct action. Cognitive Neuropsychology, 15, 645-683.

RidDOCH, M. J., \& HumphreYs, G. W. (1987). Visual object processing in optic aphasia: A case of semantic access agnosia. Cognitive Neuropsychology, 4, 131-185.

RidDOCH, M. J., HumphreYs, G. W., \& EdWARDS, M. G. (2000). Visual affordances and object selection. In S. Monsell \& J. Driver (Eds.), Attention and performance XVIII: Control of cognitive processes (pp. 603-626). Cambridge, MA: MIT Press.

Riddoch, M. J., Humphreys, G. W., Heslop, J., \& Castermans, E. (2002). Dissociations between object knowledge and everyday action. Neurocase, 8, 100-110.

Riddoch, M. J., Humphreys, G. W., \& Price, C. J. (1989). Routes to action: Evidence from apraxia. Cognitive Neuropsychology, 6, 437-454.

Roy, E. A., \& SQuare, P. A. (1985). Common considerations in the study of limb, verbal, and oral apraxia. In E. A. Roy (Ed.), Neuropsychological studies of apraxia and related disorders (pp. 112-162). Amsterdam: North-Holland.

Rumiati, R. I., \& Humphreys, G. W. (1998). Recognition by action: Dissociating visual and semantic routes to actions in normal observers. Journal of Experimental Psychology: Human Perception \& Performance, 24, 631-647.

Saenz, M., Buracas, G. T., \& Boynton, G. M. (2002). Global effects of feature-based attention in human visual cortex. Nature Neuroscience, 5, 631-632.

Simon, J. R., \& Small, A. M. (1969). Processing auditory information: Interference from an irrelevant cue. Journal of Applied Psychology, 53, 433-435.
Sirigu, A., Duhamel, J. R., Cohen, L., Pillon, B., Dubois, B., \& AGID, Y. (1996). The mental representation of hand movements after parietal cortex damage. Science, 273, 1564-1568.

Snowden, J. S., Goulding, P. J., \& Neary, D. (1989). Semantic dementia: A form of circumscribed cerebral atrophy. Behavioural Neurology, 2, 167-182.

Stroop, J. R. (1935). Studies of interference in serial verbal reactions. Journal of Experimental Psychology, 18, 643-662.

TUCKER, M., \& ElLIS, R. (1998). On the relations between seen objects and components of potential actions. Journal of Experimental Psychology: Human Perception \& Performance, 24, 830-846.

WARRINGTON, E. K. (1975). The selective impairment of semantic memory. Quarterly Journal of Experimental Psychology, 27, 187-199.

Yoon, E. Y., Heinke, D., \& Humphreys, G. W. (2002). Modelling direct constraints on action selection: The naming and action model (NAM). Visual Cognition, 9, 615-661.

Yoon, E. Y., \& HumphreYs, G. W. (2005). Dissociative effects of viewpoint and semantic priming on action and semantic decisions: Evidence for dual route to action from vision. Manuscript submitted for publication.

\section{NOTES}

1. The effect of noise within the visual route to action was particularly pronounced if the visual route had an initial, fast influence on action retrieval.

2. In this experiment, a third task was also included in which verification responses were made to a label representing a context in which the object might typically be found (is the object found in a kitchen or not?). This third condition represented an attempt to assess access to semantic knowledge without name retrieval being required - the idea being that judgments of where an object is typically found depend on semantic knowledge (see, e.g., Warrington, 1975). However, performance in this task was variable across participants and items, presumably because there is graded knowledge of whether objects are found in kitchens. Due to this variability, the data are not included here.

3. We thank Arthur Glenberg for suggesting this account. 
APPENDIX

Objects and Instructions for the Tasks

\begin{tabular}{rllll}
\hline & Kitchen Item & Action & Nonkitchen Item & Action \\
\hline 1. & butter knife & spreading & axe & chopping \\
2. & cafetiere & plunging & ball & throwing \\
3. & cocktail shaker & shaking & bell & ringing \\
4. & colander & draining & cigarette & lighting \\
5. & cup & drinking & comb & combing \\
6. & fork & eating & dustbrush & sweeping \\
7. & frying pan & flipping & fountain pen & writing \\
8. & garlic press & crushing & gun & shooting \\
9. & grater & grating & hair dryer & blowing \\
10. & ice cream scooper & scooping & hammer & hitting \\
11. & j-cloth & wiping & key & locking \\
12. & kettle & boiling & lighter & igniting \\
13. & knife & slicing & needle & sewing \\
14. & meat pounder & pounding & paintbrush & painting \\
15. & nut cracker & cracking & pencil sharpener & sharpening \\
16. & opener & opening & perfume bottle & spraying \\
17. & orange juicer & squeezing & pipe & smoking \\
18. & pepper mill & grinding & plug & connecting \\
19. & rolling pin & rolling & racket & playing \\
20. & saltshaker & sprinkling & razor & shaving \\
21. & saucepan & cooking & rubber & erasing \\
22. & scourer & rubbing & saw & cutting \\
23. & sieve & sifting & scissors & snipping \\
24. & spatula & mixing & scraper & stripping \\
25. & spoon & stirring & screwdriver & screwing \\
26. & teapot & pouring & spanner & tightening \\
27. & tongs & gripping & tambourine & rattling \\
28. & washing-up brush & scrubbing & telephone & dialing \\
29. & whisk & beating & toothbrush & brushing \\
30. & wine glass & sipping & trowel & digging \\
\hline & & & & \\
& & & & \\
& & & &
\end{tabular}

\section{Instructions}

Name verification. Your task is to verify, as quickly as you can without making errors, whether the depicted object matches the name presented with it. On some trials you will see a hand grasping the object, but this is irrelevant to the decision you will make.

Action verification. Your task is to verify, as quickly as you can without making errors, whether the depicted object would be used to make the named action that is presented with it. On some trials you will see a hand grasping the object, but this is irrelevant to the decision you will make.

(Manuscript received March 28, 2003;

revision accepted for publication October 5, 2004.) 